\title{
Evaluation of the consistency ratios of cervical smear, cervical biopsy and conization results
}

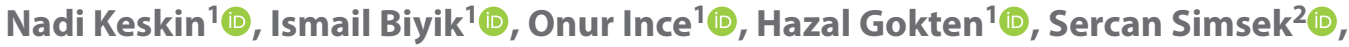 \\ Cenk Soysal ${ }^{1}$, , Ozlem Erten ${ }^{1}$ (D) \\ ${ }^{1}$ Kutahya Health Sciences University, Turkey \\ ${ }^{2}$ Kutahya Evliya Celebi Education and Research Hospital, Turkey
}

\begin{abstract}
Objectives: Possible discrepancies between the cervical smear, biopsy histology and loop electrosurgical excision procedure (LEEP) results of the same patient is a matter of debate in the literature. In this study, we investigate the degree to which these results differ, and the clinical reasons for these differences.

Material and methods: With a retrospective design, cervical smear, cervical biopsy and LEEP results of patients were compared in terms of consistency. One hundred sixty-four patients who underwent till LEEP procedure due to pathologic initial smear and biopsy results between January 2015 and March 2020 were included in the study.

Results: Exact diagnosis discrepancy and high grade squamous intraepithelial lesion (HSIL) discrepancy were $78.9 \%$ and $50.0 \%$ between smear and cervical biopsy, $64.6 \%$ and $31.7 \%$ between cervical smear and LEEP and $43.8 \%$ and $28.1 \%$ between cervical biopsy and LEEP results, respectively. Age did not affect the consistency rates of pathologic results between smear-biopsy $(p=0.408)$ and biopsy-LEEP $(p=0.590)$. However, the probability of the consistency of smear and LEEP results exhibited a statistically significant linear relation with age $(O R=1.043, p=0.015)$. HPV infections did not affect the discrepancy between smear-biopsy $(p=0.533)$, smear-LEEP $(p=1.000)$ and biopsy-LEEP $(p=0.529)$.

Conclusions: Smear technique has a serious discrepancy and under-diagnosis problem when its results are compared with biopsy and LEEP. The consistency between smear and LEEP results appears to improve with age. When HSIL is evaluated in terms of detection, this discrepancy decreases. A smear test can detect HSIL and carcinoma with a higher accuracy than low-grade lesions.
\end{abstract}

Key words: cervical smear; cervical biopsy; LEEP; conization; discrepancy

Ginekologia Polska 2021; 92, 11: 778-783

\section{INTRODUCTION}

Cervical cancer is a type of gynaecological cancer that turns into an invasive lesion following the premalignant lesion stage. The incidence of cervical cancer in Europe was $11.2 / 100,000$ and the mortality rate was $3.8 / 100,000$ women according to a 2018 study [1]. Premalignant lesions are cancer precursors that occur due to dysplasia in the cervical epithelium. With cervical screening programs, premalignant lesions can be detected before they turn into invasive cancer.

Cervical Pap smear screening, which was defined by Papanicolaou in the 1940s, is widely used in the world today [2]. Cervical screening programs are based on cervical cancer precursors and early-stage disease detection. Screening programs provide the opportunity to catch and treat the disease at an early stage or the premalignant stage. However, there is a risk of unnecessary cervical intervention due to false positivity or a possible cervical cancer bypasses due to false negativity. The sensitivity of Pap smear was reported as $<70 \%$ in some studies [3-5]. A recent study reported that the sensitivity, specificity, positive predictive and negative predictive values of Pap smear were $55.5 \%, 75 \%, 88.2 \%$ and $33.3 \%$, respectively [6].

There can be discrepancies between cervical cytology results and biopsy histology. Discrepancy rates were reported between $11-28 \%$ in various studies [7-9]. Similarly, studies are reporting $43-86 \%$ consistency between

\section{Corresponding author:}

Nadi Keskin

Kütahya Sağlık Bilimleri Üniversitesi Evliya Çelebi Yerleşkesi Tavşanlı Yolu 10. km 43100 Kütahya, Turkey

e-mail: nadikeskin@gmail.com 
colposcopy-guided cervical biopsy results and LEEP results [10-14].

In this study, we aim to investigate the discrepancy rates between cervical smear, cervical biopsy and conization results and the clinical reasons behind them.

\section{MATERIAL AND METHODS}

This diagnostic test comparison study was conducted with permission from the Clinical Research Ethics Committee of Kutahya Health Sciences University in Kutahya, Turkey (2020/07-13). Cervical cytology, cervical biopsy and LEEP results of patients who underwent LEEP between January 2015 and March 2020 at Evliya Çelebi Education and Research Hospital of Kutahya Health Sciences University were compared in terms of consistency. Demographic information, clinical and pathology records of patients were obtained from patient files. 164 cases were included in the study.

Thin prep cytologic test (TCT, Hologic, USA) was used to perform the liquid-based cervical cytology. Pap smear results were classified according to the Bethesda system as "negative in terms of malignancy and intraepithelial lesion", "atypical squamous cells of undetermined significance (ASC-US)", "low grade squamous intraepithelial lesion (LSIL)” and "high grade squamous intraepithelial lesion (HSIL)". In the cervical biopsy, intraepithelial lesions were classified as cervical intraepithelial neoplasia (CIN) I, II, III based on the degree of dysplasia [15]. Abnormal Pap smear results were managed based on the ASCCP recommendations in the hospital where the study took place [15].

For women 30 years old or older with HPV-positive but cytology-negative co-testing, repeat co-testing was done one year later. If the HPV test was positive or cytology was ASC-US or worse at the one-year repeat co-test, colposcopy was performed. The colposcopy indications were performed according to The American Society for Colposcopy and Cervical Pathology Colposcopy Standards: abnormal or inconclusive PAP smear test, abnormality found during pelvic examination, abnormal genital tract bleeding, or unexplained cervicovaginal discharge and past cytologic and/or pathologic anogenital tract abnormalities [16]. As a result of cervical cytology, a biopsy was performed in the case of abnormal findings and/or abnormal findings in colposcopic cervical examination [17]. In cases where a colposcopic examination was insufficient, LEEP was applied to the non-pregnant cases. HPV results of 127 (77.4\%) subjects were evaluated.

Following the ASCCP recommendations, patients who were not pregnant and who had HSIL as a result of cervical cytology, except for the 21-24 age group, underwent direct LEEP without or after a colposcopic directed biopsy.

\section{Statistical analyses}

Clinical and pathological data of the cases were analyzed. Consistency of the patients' results for smear, colposcopy-directed biopsy and LEEP were presented by pairwise comparison tables and consistency rates were calculated by using Cohen's kappa ( $\mathrm{k}$ ) coefficient. In addition, in order to detect the discrepancy due to under-diagnosis, weighted Cohen's kappa model was used to give penalty only for under-diagnosis of the former technique compared to the latter technique's pathology result. In this analysis the pathology results with same diagnosis or over-diagnosis were counted as consistency. $\mathrm{k}$ values $<0$ were considered as no agreement, $0-0.20$ as slight, $0.21-0.40$ as fair, $0.41-0.60$ as moderate, $0.61-0.80$ as substantial, and $0.81-1.00$ as almost perfect agreement. In order to evaluate the effect of age on techniques' consistency, generalized additive model (GAM) with restricted maximum likelihood (REML) approach was performed. In the analysis of consistency of smear and LEEP techniques, GAM analysis gave a smooth with one degree of freedom which indicated that the relation was linear. Therefore, logistic regression analysis was performed to evaluate the probability of consistency between smear and LEEP. Pearson chi square test was conducted in order to evaluate the consistency between techniques in patients with and without initial HPV positivity. All statistical analyses were performed using SPSS statistical software (version 25.0; SPSS Inc., Chicago, IL, USA) and R statistical computing software (version 3.5.0, https://www.r-project.org/). A p-value $<0.05$ was considered as statistically significant.

\section{RESULTS}

The study was conducted with 164 cases. The mean age of the patients was $46.68 \pm 9.56$ years. There were no pregnant women in the sample. A colposcopic cervical biopsy was performed in 128 (78\%) of the 164 patients who underwent LEEP, and the remaining 36 (22\%) patients underwent LEEP directly without a colposcopic biopsy. One hundred twenty-seven cases had HPV test results. High-risk HPV types were positive in 115 (90.4\%) cases, low-risk HPV types were positive in six (4.8\%) cases and negative in six (4.8\%) cases. 16 , $18,26,31,33,35,39,45,51,52,53,56,58,59,66,68,73$ and 83 were accepted as high-risk HPV types. 6, 11, 40, 42, 43, $44,54,61,70,72$ and 81 were accepted as low risk HPV types.

Agreement level between the techniques on the exact diagnosis are presented in the first column of Table 1 with Cohen Kappa statistics. In the second column, weighted Cohen's Kappa statistics measuring the reliability of the pairwise comparison of the techniques are given. These weighted kappa values accepted only the under-diagnosed results of the first technique compared to the latter as source of discrepancy. The exact diagnosed and over-diagnosed 
Table 1. Consistency of patients' pathologic results between smear colposcopy-directed biopsy and loop electrosurgical excision procedure were presented by pairwise comparison tables and consistency rates

\begin{tabular}{|l|l|l|l|l|l|l|l|l|l|}
\hline & \multicolumn{3}{|l}{ Exact Diagnosis } & \multicolumn{3}{l|}{ Under-diagnosis Discrepancy } & \multicolumn{3}{l|}{ HSIL vs non-HSIL } \\
\cline { 2 - 10 } & Cohen's K & p value & Discrepancy & $\begin{array}{l}\text { Weighted } \\
\text { Cohen's Ka }\end{array}$ & p value & Discrepancy & Cohen's K & p value & Discrepancy \\
\hline $\begin{array}{l}\text { Smear vs Colposcopic } \\
\text { biopsy }\end{array}$ & -0.001 & 0.962 & $\begin{array}{l}78.9 \% \\
(101 / 128)\end{array}$ & -0.021 & 0.873 & $\begin{array}{l}75.0 \% \\
(96 / 128)\end{array}$ & 0.027 & 0.520 & $\begin{array}{l}50.0 \% \\
(64 / 128)\end{array}$ \\
\hline Smear vs LEEP & 0.067 & 0.075 & $\begin{array}{l}64.6 \% \\
(106 / 164)\end{array}$ & 0.010 & 0.931 & $\begin{array}{l}60.4 \% \\
(99 / 164)\end{array}$ & 0.170 & $<0.001$ & $\begin{array}{l}31.7 \% \\
(52 / 164)\end{array}$ \\
\hline $\begin{array}{l}\text { Colposcopic biopsy } \\
\text { vs LEEP }\end{array}$ & 0.310 & $<0.001$ & $\begin{array}{l}43.8 \% \\
(56 / 128)\end{array}$ & 0.360 & 0.064 & $\begin{array}{l}17.2 \% \\
(22 / 128)\end{array}$ & 0.440 & $<0.001$ & $\begin{array}{l}28.1 \% \\
(36 / 128)\end{array}$ \\
\hline
\end{tabular}

${ }^{a}$ No penalty were given to the over-diagnosed results of the former technique compared to latter technique; LEEP — loop electrosurgical excision procedure

\begin{tabular}{l}
$\begin{array}{l}\text { Table 2. Comparison of pathological results between a cervical smear and colposcopy- } \\
\text { directed biopsy of patients }\end{array}$ \\
\hline
\end{tabular}

\begin{tabular}{|l|l|}
\hline $75.00 \%$ & Under-diagnosed $(n=96)$ \\
\hline $21.1 \%$ & Correctly diagnosed $(n=27)$ \\
\hline $3.9 \%$ & Over-diagnosed $(n=5)$ \\
\hline
\end{tabular}

LSIL — low grade squamous intraepithelial lesion; HSIL — high grade squamous intraepithelial lesion

\begin{tabular}{l}
$\begin{array}{l}\text { Table 3. Comparison of pathological results between cervical colposcopy-directed biopsy } \\
\text { and loop electrosurgical excision procedure of patients }\end{array}$ \\
\hline \\
\hline
\end{tabular}

\begin{tabular}{|l|l|}
\hline $17.20 \%$ & Under-diagnosed $(n=22)$ \\
\hline $56.20 \%$ & Correctly diagnosed $(n=72)$ \\
\hline $4.30 \%$ & Over-diagnosed $(n=34)$ \\
\hline
\end{tabular}

LEEP — loop electrosurgical excision procedure; LSIL — low grade squamous intraepithelial lesion; HSIL — high grade squamous intraepithelial lesion

results of the prior technique with reference to the latter technique were accepted as agreement. Lastly, all pathology results were recoded as being $\mathrm{HSIL}+$ or not, and the consistency on HSIL agreement was presented with again Cohen's Kappa statistic.

Pairwise comparisons of cervical smear, colposcopy-directed biopsy and LEEP results are given in Table 2, 3 and 4 .
According to GAM analysis which evaluates not only linear but also non-linear relations, age did not appear to affect the consistency of smear-biopsy $(p=0.408)$ and biopsy-LEEP results $(p=0.590)$. However, age demonstrated a statistically significant smooth $(p=0.016)$ with one degree of freedom (which implies a linear relation as shown in Figure 1), on its effect on the consistency rates between the smear and 


\begin{tabular}{|c|c|c|c|c|c|}
\hline & \multicolumn{4}{|l|}{ LEEP } & \multirow[b]{2}{*}{ TOTAL } \\
\hline & $\begin{array}{l}\text { No } \\
\text { Dysplasia }\end{array}$ & LSIL & HSIL & Carcinoma & \\
\hline \multicolumn{6}{|l|}{ Smear } \\
\hline No Dysplasia & $43(32.8 \%)$ & 48 (36.6\%) & $38(29.0 \%)$ & $2(1.5 \%)$ & 131 \\
\hline LSIL & $5(22.7 \%)$ & $6(27.3 \%)$ & $11(50.0 \%)$ & $0(0.0 \%)$ & 22 \\
\hline HSIL & $1(9.1 \%)$ & $1(9.1 \%)$ & $9(81.8 \%)$ & $0(0.0 \%)$ & 11 \\
\hline Carcinoma & $0(0.0 \%)$ & $0(0.0 \%)$ & $0(0.0 \%)$ & $0(0.0 \%)$ & 0 \\
\hline TOTAL & 49 & 55 & 58 & 2 & 164 \\
\hline
\end{tabular}

\begin{tabular}{|l|l|}
\hline $60.4 \%$ & Under-diagnosed $(n=99)$ \\
\hline $35.4 \%$ & Correctly diagnosed $(n=58)$ \\
\hline $4.3 \%$ & Over-diagnosed $(n=7)$ \\
\hline
\end{tabular}

LEEP — loop electrosurgical excision procedure; LSIL — low grade squamous intraepithelial lesion; HSIL — high grade squamous intraepithelial lesion

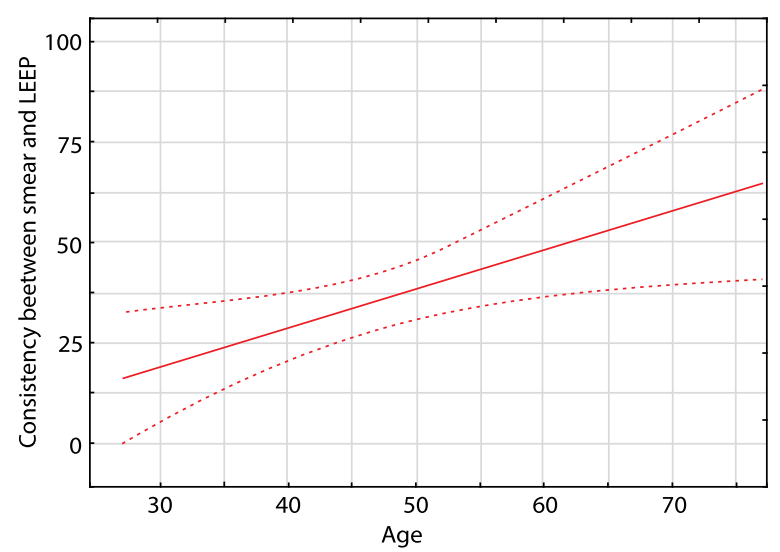

Figure 1. Diagnostic consistency of cervical biopsy and loop electrosurgical excision procedure (LEEP) results according to age

LEEP. The consistency rates increased with the odds ratio of 1.043 by one-year increase in age $(p=0.016)$.

Between HPV + and HPV - patients, the consistency rates of smear-biopsy comparison $(23 / 104,0 / 5, p=0.533$, respectively), biopsy-LEEP comparison (59/104, 3/5, $p=1.000$, respectively) and smear-LEEP comparison (35/120, 3/6, $p=0.529$, respectively) were all statistically similar.

\section{DISCUSSION}

In this study, the discrepancy was determined as $78.9 \%$ between a cervical smear and cervical biopsy results, $64.6 \%$ between cervical smear and LEEP results, and $43.8 \%$ between colposcopic biopsy and LEEP results. Increasing age appears to have a positive and linear effect on the consistency rates between smear and LEEP. In detecting the HSIL accurately, there were $50.0 \%$ discrepancy between a cervical smear and cervical biopsy results, 31.7\% between cervical smear and LEEP results, and $28.1 \%$ between colposcopic biopsy and LEEP results.
There are several studies investigating the consistency of smear and colposcopic biopsy techniques in the literature. In the Anschau et al. [18] study, in 54\% (235/431) of the cases there was concordance between cytology and biopsy histology of the cervix. In 34.5\% (149/431) of cases, cytology pointed to a less severe diagnosis compared to histology. In 10.9\% (47/431) of the cases, cytology indicated a more severe diagnosis. In our study, smear-cervical biopsy concordance was found to be $21.1 \%$. Like Anschau's study, for smear results, we found a much higher rate of under-diagnosis (75\%) than over-diagnosis (3.9\%). In the Pap smear test, Goodman et al. [19] found that $5 \%$ of the women who underwent co-testing with negative Pap tests had HSIL on the follow-up biopsy. In our study, 47.6\% (49/103) of the women with negative smear test had HSIL on cervical biopsy. These results suggest that the smear technique has a serious discrepancy and under-diagnosis problem relative to biopsy and LEEP.

Poomtavorn et al. [20], report the rate of colposcopic cervical biopsy discrepancy as $45.7 \%$ with the Pap smear test and the rate of colposcopic directed biopsy histology discrepancy as $29.5 \%$ with the Pap smear test. Alanbay et al. [21], report that when the level of atypia increases in cervical cytology, cytology-histology consistency rate increases. In our study, while the exact diagnosis of cervical cytology with LEEP was $27.3 \%$ for LSIL, this rate increased to $81.8 \%$ for HSIL. These results are consistent with the hypothesis in Alanbay et al. [21].

Several demographic parameters and clinical features were investigated as the possible drivers of the discrepancy in the results of the different techniques. Poomtavorn et al. [20], report that factors associated with cytohistologic discrepancy were nulliparity and postmenopausal status and having no oral contraceptive pill use. In addition, Jung et al., report that the concordance between cervical punch 
biopsy and conization is lower between the ages of 30 to 60 , and higher after the age of 60 compared to women before the age of 30 . By the way, these comparisons were statistically insignificant, however, which suggests a complex relationship between age and the concordance of techniques [14]. Keeping this complex relation in mind, we used GAM analysis which does not presume a linear relationship between parameters. Nevertheless, our analysis has pointed out a statistically significant linear relationship between increasing age and the consistency rates between smear and LEEP (Fig. 1). The reason behind the discrepancy of the results at younger ages may be due to the difficulty of evaluating the pathologic smear results, since women at the reproductive stage have higher infection rates.

The literature indicates less discrepancy between cervical biopsy and conization results relative to smear and conization. Aydogmus et al. [22], report a 36\% discrepancy between cervical biopsy and conization results. The prospective study of Peousis et al. [23], compares the results of colposcopy, punch biopsy and conization and report the discrepancy between cervical biopsy and conization results accompanied by colposcopy as $36.6 \%$ (38/104). In our study, a discrepancy rate of $17.2 \%(22 / 128)$ was found between colposcopic biopsy and LEEP results. In studies evaluating the presence of HSIL, there is evidence of low concordance between colposcopic cervical biopsy and LEEP results $[13,14]$. A prospective study shows that colposcopic biopsy results report lower rates of HSIL than LEEP [24]. In the study of Kim and et al. [24], in terms of HPV detection, $67.7 \%$ concordance was found between colposcopic biopsy and LEEP results and $26.6 \%$ of the colposcopic biopsy results was under-diagnosis. In the same study, it was emphasized that in various studies that it is difficult to differentiate LSIL and HSIL with colposcopic cervical biopsy, and therefore pathologists use immune-histochemical staining for cases in-between $[25,26]$. In addition, like our results, all these studies indicate that the consistency between smear and histologic results increases for more invasive lesions of the cervix [19, 23-27].

The reason behind the discrepancy of the results between techniques may be due to performing incorrect technique during the sampling or misinterpretation of the results of the techniques. In several studies, it is reported that approximately two-thirds of the results that were false negative due to cervical cytology resulted from insufficient sampling [28].

In the literature, there are few studies on HPV and its relationship with the discrepancy of the techniques. In one study, it is reported that HPV testing and genotyping had limited value in risk stratification due to the extremely low positive predictive value and that focused rescreening of
hrHPV-positive NILM with obscuring factors may help reduce the interpretation variances.

This study is the one of the few in the literature which evaluates the pairwise agreement of the techniques. The under-diagnosis discrepancy was also specifically investigated. The positive linear relationship between increasing age and the consistency rate of the smear and LEEP was presented for the first time with no statistical presumptions of linearity. However, the major limitation of the study is that the analysis regarding the discrepancy and HPV state may suffer from type 2 error since the sample is small. One hundred twenty-seven of the cases had HPV results recorded and HPV negative cases consisted only $4.8 \%$ of this sample. The low share of HPV negative cases is expected since the premalignant lesions of the cervix are mainly caused by the HPV infections.

\section{CONCLUSIONS}

Smear technique has a serious discrepancy and under-diagnosis problem when its results are compared with biopsy and LEEP. Increasing age seems to improve consistency between smear and LEEP results. When HSIL is evaluated in terms of detection, the discrepancy rate decreases. A smear test can detect HSIL and carcinoma with higher accuracy than low-grade lesions.

\section{Conflict of interest}

No authors have any conflict-of-interest report.

\section{REFERENCES}

1. Bruni L, Albero G, Serrano B, et al. ICO/IARC information centre on HPV and cancer (HPV information centre). Human papillomavirus and related diseases in the world. Summary report. https://www. hpvcentre. net/statistics/reports/XWX.pdf (19.07.2019).

2. Papanicolaou GN, Traut HF. The diagnostic value of vaginal smears in carcinoma of the uterus. Am J Obstet Gynecol. 1941; 42: 193.

3. Bhattacharyya AK, Nath JD, Deka H. Comparative study between pap smear and visual inspection with acetic acid (via) in screening of CIN and early cervical cancer. J Midlife Health. 2015; 6(2): 53-58, doi: 10.4103/0976-7800.158942, indexed in Pubmed: 26167054.

4. Bobdey S, Balasubramanium G, Kumar A, et al. Cancer Screening: Should Cancer Screening be Essential Component of Primary Health Care in Developing Countries? Int J Prev Med. 2015; 6: 56, doi: 10.4103/20087802.160053, indexed in Pubmed: 26236443.

5. Karimi-Zarchi M, Zanbagh L, Shafii A, et al. Comparison of Pap Smear and Colposcopy in Screening for Cervical Cancer in Patients with Secondary Immunodeficiency. Electron Physician. 2015; 7(7): 1542-1548, doi: 10.19082/1542, indexed in Pubmed: 26767111.

6. Nkwabong $E$, Laure Bessi Badjan I, Sando Z. Pap smear accuracy for the diagnosis of cervical precancerous lesions. Trop Doct. 2019; 49(1): 34-39, doi: 10.1177/0049475518798532, indexed in Pubmed: 30222058.

7. Ibrahim SN, Krigman HR, Coogan AC, et al. Prospective correlation of cervicovaginal cytologic and histologic specimens. Am J Clin Pathol. 1996; 106(3): 319-324, doi: 10.1093/ajcp/106.3.319, indexed in Pubmed: 8816588.

8. Tritz DM, Weeks JA, Spires SE, et al. Etiologies for non-correlating cervical cytologies and biopsies. Am J Clin Pathol. 1995; 103(5): 594-597, doi: 10.1093/ajcp/103.5.594, indexed in Pubmed: 7741105.

9. Bewtra C, Pathan M, Hashish $\mathrm{H}$. Abnormal Pap smears with negative follow-up biopsies: improving cytohistologic correlations. Diagn 
Cytopathol. 2003; 29(4): 200-202, doi: 10.1002/dc.10329, indexed in Pubmed: 14506671.

10. Chappatte OA, Byrne DL, Raju KS, et al. Histological differences between colposcopic-directed biopsy and loop excision of the transformation zone (LETZ): a cause for concern. Gynecol Oncol. 1991; 43(1): 46-50, doi: 10.1016/0090-8258(91)90007-r, indexed in Pubmed: 1959787.

11. Duesing N, Schwarz J, Choschzick M, et al. Assessment of cervical intraepithelial neoplasia (CIN) with colposcopic biopsy and efficacy of loop electrosurgical excision procedure (LEEP). Arch Gynecol Obstet. 2012; 286(6): 1549-1554, doi: 10.1007/s00404-012-2493-1, indexed in Pubmed: 22865036.

12. Witt BL, Factor RE, Jarboe EA, et al. Negative loop electrosurgical cone biopsy finding following a biopsy diagnosis of high-grade squamous intraepithelial lesion: frequency and clinical significance. Arch Pathol Lab Med. 2012; 136(10): 1259-1261, doi: 10.5858/arpa.2011-0494-OA, indexed in Pubmed: 23020732.

13. Kabaca C, Koleli I, Sariibrahim B, et al. Is cervical punch biopsy enough for the management of low-grade cervical intraepithelial neoplasia? J Low Genit Tract Dis. 2014; 18(3): 240-245, doi: 10.1097/LGT.0b013e3182aa08f6, indexed in Pubmed: 24633166.

14. Jung $Y$, Lee AhRa, Lee SJ, et al. Clinical factors that affect diagnostic discrepancy between colposcopically directed biopsies and loop electrosurgical excision procedure conization of the uterine cervix. Obstet Gynecol Sci. 2018; 61(4): 477-488, doi: 10.5468/ogs.2018.61.4.477, indexed in Pubmed: 30018902.

15. Massad LS, Einstein MH, Huh WK, et al. 2012 ASCCP Consensus Guidelines Conference, 2012 ASCCP Consensus Guidelines Conference. 2012 updated consensus guidelines for the management of abnormal cervical cancer screening tests and cancer precursors. J Low Genit Tract Dis. 2013; 17(5 Suppl 1): S1-S27, doi: 10.1097/LGT.0b013e318287d329, indexed in Pubmed: 23519301.

16. Khan MJ, Werner CL, Darragh TM, et al. ASCCP Colposcopy Standards: Role of Colposcopy, Benefits, Potential Harms, and Terminology for Colposcopic Practice. J Low Genit Tract Dis. 2017; 21(4): 223-229, doi: 10.1097/LGT.0000000000000338, indexed in Pubmed: 28953110.

17. Kim TH, Han JH, Shin E, et al. Clinical Implication of p16, Ki-67, and Proliferating Cell Nuclear Antigen Expression in Cervical Neoplasia: Improvement of Diagnostic Accuracy for High-grade Squamous Intraepithelial Lesion and Prediction of Resection Margin Involvement on Conization Specimen. J Cancer Prev. 2015; 20(1): 70-77, doi: 10.15430/JCP.2015.20.1.70, indexed in Pubmed: 25853106.

18. Anschau F, Guimarães Gonçalves MA. Discordance between cytology and biopsy histology of the cervix: what to consider and what to do.
Acta Cytol. 2011; 55(2): 158-162, doi: 10.1159/000320911, indexed in Pubmed: 21325800.

19. Goodman S, Mody RR, Coffey D, et al. Negative Pap tests in women with high-grade cervical lesions on follow-up biopsies: Contributing factors and role of human papillomavirus genotyping. Diagn Cytopathol. 2018; 46(3): 239-243, doi: 10.1002/dc.23874, indexed in Pubmed: 29230975.

20. Poomtavorn Y, Himakhun W, Suwannarurk K, et al. Cytohistologic discrepancy of high-grade squamous intraepithelial lesions in Papanicolaou smears. Asian Pac J Cancer Prev. 2013; 14(1): 599-602, doi: 10.7314/apjcp.2013.14.1.599, indexed in Pubmed: 23534802.

21. Alanbay I, Öztürk M, Fıratlıgil FB, et al. Cytohistological discrepancies of cervico-vaginal smears and HPV status. Ginekol Pol. 2017; 88(5): 235-238, doi: 10.5603/GP.a2017.0044, indexed in Pubmed: 28580567.

22. Aydogmus $\mathrm{H}$, Sen S, Aydogmus S. Pathological discrepancy between colposcopic directed cervical biopsy and conisation results: A five years experience of a single center in Turkey. Pak J Med Sci. 2019; 35(6): 1627-1630, doi: 10.12669/pjms.35.6.408, indexed in Pubmed: 31777505.

23. Petousis S, Christidis P, Margioula-Siarkou C, et al. Discrepancy between colposcopy, punch biopsy and final histology of cone specimen: a prospective study. Arch Gynecol Obstet. 2018; 297(5): 1271-1275, doi: 10.1007/s00404-018-4714-8, indexed in Pubmed: 29442140.

24. Kim Selk, Kim SeJ, Suh DH, et al. Pathologic discrepancies between colposcopy-directed biopsy and loop electrosurgical excision procedure of the uterine cervix in women with cytologic high-grade squamous intraepithelial lesions. J Gynecol Oncol. 2020; 31(2): e13, doi: 10.3802/jgo.2020.31.e13, indexed in Pubmed: 31912671.

25. Dalla Palma P, Giorgi Rossi P, Collina G, et al. NTCC Pathology Group. The reproducibility of CIN diagnoses among different pathologists: data from histology reviews from a multicenter randomized study. Am J Clin Pathol. 2009; 132(1): 125-132, doi: 10.1309/AJCPBRK7D1YIUWFP, indexed in Pubmed: 19864243.

26. Martin CM, O'Leary JJ. Histology of cervical intraepithelial neoplasia and the role of biomarkers. Best Pract Res Clin Obstet Gynaecol. 2011; 25(5): 605-615, doi: 10.1016/j.bpobgyn.2011.04.005, indexed in Pubmed: 21636328.

27. Byrom J, Douce G, Jones PW, et al. Should punch biopsies be used when high-grade disease is suspected at initial colposcopic assessment? A prospective study. Int J Gynecol Cancer. 2006; 16(1): 253-256, doi: 10.1111/j.1525-1438.2006.00344.x, indexed in Pubmed: 16445640.

28. Vooijs GP, Elias A, van der Graaf Y, et al. The influence of sample takers on the cellular composition of cervical smears. Acta Cytol. 1986; 30(3): 251-257, indexed in Pubmed: 3459325. 